\title{
Platelet-derived growth factor signaling in human malignancies
}

\author{
Kun-Wei $\mathrm{Liu}^{1,2 *}$, Bo Hu${ }^{1,3}$ and Shi-Yuan Cheng ${ }^{1,2}$
}

\begin{abstract}
Platelet-derived growth factors (PDGFs) and their receptors were identified and purified decades ago. PDGFs are important during normal development and in human cancers. In particular, autocrine PDGF signaling has been implicated in various types of malignancies such as gliomas and leukemia. In contrast, paracrine signaling was found in cancers that originate from epithelial cells, where it may be involved in stromal cell recruitment, metastasis, and epithelial-mesenchymal transition. This editorial briefly discusses autocrine and paracrine PDGF signaling and their roles in human cancers, and introduces a series of review articles in this issue that address the possible roles of PDGFs in various processes involved in different types of cancers.
\end{abstract}

Key words Platelet-derived growth factor, cancer, tumor progression, drug resistance

Platelet-derived growth factor (PDGF) was first identified and purified from the platelets that stimulate proliferation of fibroblasts, smooth muscle cells, and glial cells ${ }^{[1-3]}$. Subsequent studies showed that the $B$ chain of PDGF (PDGF-B) was closely related to the oncogene $v$-sis of simian sarcoma virus (SSV) and that autocrine PDGF-B signaling was sufficient for malignant transformation mediated by SSV ${ }^{[4,5]}$. These findings provided a paradigm describing how dysregulation of normal growth control machinery leads to malignant transformation.

Autocrine PDGF signaling has been implicated in gliomas ${ }^{[6]}$, sarcomas ${ }^{[7]}$, and breast cancers ${ }^{[8]}$. PDGF confers proliferation, survival, and tissue metastasis advantages to cancer cells and promotes angiogenesis from surrounding vasculatures. In several types of epithelial cancers such as breast cancer, autocrine PDGF signaling contributes to epithelial-mesenchymal transition (EMT) and tumor metastasis ${ }^{[9,10]}$. Paracrine

\footnotetext{
Authors' Affiliations: 'University of Pittsburgh Cancer Institute, Departments of ${ }^{2}$ Pathology and ${ }^{3}$ Medicine, University of Pittsburgh School of Medicine, Pittsburgh, PA 15213, USA

* Current address: Tumor Development Program, Sanford-Burnham Medical Research Institute, 10901 North Torrey Pines Road, La Jolla, CA 92037-1062, USA

Corresponding Authors:

Bo Hu, Cancer Institute \& Department of Medicine, University of Pittsburgh School of Medicine, Pittburgh, PA 15213, USA. Tel: +1-412-6237791; Fax: +1-412-623-4840; Email: hub@upmc.edu.

Shi-Yuan Cheng, Cancer Institute \& Department of Pathology, University of Pittsburgh School of Medicine, Pittburgh, PA 15213, USA. Tel: +1412-623-3261; Fax: +1-412-623-4840; Email: chengs@ upmc.edu. dol: $10.5732 /$ cjc. 011.10300
}

PDGF signaling may play a role in malignant transformation by recruiting different types of stromal cells to the tumor mass. These stromal cells can then support tumor growth and survival, and promote tumor angiogenesis and metastasis ${ }^{[11,12]}$. Moreover, PDGF signaling in the tumor stroma contributes to the increased interstitial fluid pressure (IFP) observed in most solid tumors, implying that inhibition of PDGF signaling may improve the efficacy of chemotherapies ${ }^{[11]}$. This editorial provides an overview of previous studies of PDGF signaling in human cancers and aims to prepare the readers for the review articles published in this issue of the Chinese Journal of Cancer.

\section{Activating Mutations of PDGFR and Autocrine PDGF Signaling in Tumor Progression}

One of the best examples of how autocrine PDGF signaling contributes to malignancy is from studies of malignant gliomas. Co-expression of PDGF $\alpha$-receptor $(P D G F R \propto)$ and PDGF ligands are common in glioblastoma multiforme $(\mathrm{GBM})^{[6,13]}$ and frequently associated with p53 loss ${ }^{[14]}$. Enforced expression of PDGFR $\propto$ and PDGF-A confers growth advantages to mouse and human glioma cells in the brain of immunodeficient mice ${ }^{[15]}$. Activating mutations of PDGFR $\propto$ have also been described ${ }^{[16,17]}$. The role of PDGF signaling in normal central nervous system (CNS) development and human gliomas will be discussed in the first review article of this issue ${ }^{[18]}$.

In addition to its role in gliomas, autocrine PDGF 
signaling is also involved in the development of soft tissue sarcomas. Because PDGF is a potent mitogen for various types of mesenchymal cells including fibroblasts and myofibroblasts, several studies using mouse models have been carried out to determine if PDGF overexpression can induce a malignant phenotype in these cells. As expected, a coding region in the PDGF-B gene corresponding to the $v$-sis oncogene was capable of inducing fibrosarcomas at the site of inoculation in newborn mice ${ }^{[19]}$. Subsequent studies showed that PDGFRs and PDGFs are expressed in various types of soft tissue tumors ${ }^{[7,20,21]}$, suggesting a critical role for autocrine PDGF signaling in the development of these tumors.

Autocrine PDGF signaling has also been implicated during EMT in human breast cancer ${ }^{[12]}$. EMT is a process of switching polarized epithelial cells to a migratory mesenchymal type of cells, which are largely responsible for the progression and metastasis of human cancers that originate from epithelial cells. Studies involving the EMT process in breast cancers are discussed in the second review article in this issue ${ }^{[2]}$. Components of the PDGF signaling pathway are up-regulated during transforming growth factor- $\beta$ (TGF- $\beta$ )-induced EMT in breast cancer ${ }^{[9]}$. This observation is corroborated by studies demonstrating that autocrine PDGF signaling maintains EMT and promotes metastasis in mouse mammary carcinomas ${ }^{[10]}$. Interestingly, in gliomas, high TGF- $\beta$ signaling confers poor prognosis in patients and promotes glioma cell proliferation by activating PDGFB/PDGFR signaling ${ }^{[23]}$, suggesting an evolutionarily conserved mechanism of regulating PDGF signaling in different types of human cancers.

Constitutive autocrine PDGF signaling can also be achieved by mutational activation of the genes encoding PDGF receptors or ligands. For example, a gene translocation event occurs in dermatofibrosarcoma protuberans (DFSP), a rare form of dermal sarcoma ${ }^{[24]}$ resulting in a fusion gene between collagen 1A1 (COL1A1) and PDGF-B. The COL1A1/PDGF-B fusion protein over-produces PDGF-B and creates an autocrine loop that transforms NIH3T3 cells in vitro and in vivo ${ }^{[2,26]}$. Gene fusions involving PDGFRs have also been found in other human cancers. A subgroup of chronic myeloid leukemia without $B C R-A B L$ fusion gene harbors a translocation-mediated fusion between $B C R$ gene and $P D G F R A$ gene, leading to over-activation of PDGFR $\alpha^{[27]}$. Gene fusion between Flip-like 1 (FIP1L1) and PDGFRA is also found in patients with hypereosinophilic (HES) symdrome ${ }^{[28]}$, resulting in a fusion protein with constitutive tyrosine kinase activity and transforming capacity. Consequently, these HES patients often develop acute myeloid leukemia (AML). On the other hand, the PDGFRB gene is also a target of gene translocation-mediated activation. Gene fusion events between PDGFRB and the transcription factor TEL/ETV6 as well as several other fusion partners take place in patients with chronic myeloid leukemia ${ }^{[11,12,29]}$. Additionally, a subset of gastrointestinal stromal tumors (GIST) with wild-type c-Kit often harbor intragenic mutations in PDGFRA, leading to a ligand-independent activation of the receptor and its downstream signaling pathways ${ }^{[30,31]}$. Lastly, gene mutations that constitutively activate PDGFR $\propto$ are found in malignant human gliomas, which are discussed in the following review article of this issue ${ }^{[18]}$.

\section{Paracrine PDGF Signaling in Recruiting Tumor Stroma cells and Resistance to Therapies}

PDGF signaling not only plays an essential role in promoting tumor malignancy but also contributes to the processes of tumor stromal recruitment, angiogenesis, and drug resistance ${ }^{[11]}$. Prevalent PDGFR $\alpha$ expression has been found in the stromal compartments of various types of solid tumors. PDGF ligands expressed by the neoplastic components of the tumor mass stimulate the recruitment of surrounding non-neoplastic stromal cells expressing PDGFR, including endothelial cells, pericytes, and fibroblasts, to the tumor ${ }^{[12]}$. In mouse fibrosarcomas ${ }^{[32]}$, gliomas $^{[33]}$, and melanomas ${ }^{[34]}$, paracrine PDGF/PDGFR $\beta$ signaling enhances pericyte recruitment to the tumor vasculature, thereby promoting tumor cell growth, survival, and vessel stabilization. In gliomas, paracrine PDGF signaling induces neo-angiogenesis by directly stimulating proliferation of PDGFR $\beta$-positive tumor endothelial cells ${ }^{[8,35]}$. These studies suggest that inhibition of PDGF signaling by pharmacologic intervention can potentially suppress growth and survival of both tumor and tumor-associated stromal and endothelial cells ${ }^{[36,37]}$.

PDGF signaling also supports tumor metastasis to the bone. Using an experimental prostate cancer mouse model, researchers demonstrated that blocking PDGFR thwarted the angiogenesis of tumors that had metastasized to the bone ${ }^{[38]}$. Additionally, as discussed in the third review article in this issue, PDGFR $\propto$ signaling induces prostate cancer cell metastasis to the bone ${ }^{[39]}$, further demonstrating that paracrine PDGF signaling enhances tumor growth, angiogenesis, and metastasis.

Another important component of the tumor stroma is fibroblasts ${ }^{[12]}$. Previous data suggests that recruited tumor fibroblasts are an integral part of various types of solid tumors and influence malignant transformation ${ }^{[40]}$, angiogenesis ${ }^{[41]}$, and tumor cell proliferation ${ }^{[42]}$. Therefore, blocking PDGF signaling in tumor stroma is effective in suppressing tumor growth in experimental tumor models ${ }^{[12]}$. Furthermore, the benefit of PDGF inhibition in tumor stroma could extend beyond suppression of tumor growth, angiogenesis, and metastasis. Some solid tumors frequently show high IFP, which restricts fluid convection rate across the capillary walls ${ }^{[12]}$ and results in 
low drug uptake and tumor resistance to cytotoxic drug treatments. One of the factors that contributes to high IFP within the tumor mass is PDGFR ${ }^{[43]}$. Indeed, inhibition of PDGFR signaling by imatinib reduces tumor IFP and enhances the drug uptake into tumor cells ${ }^{[44,45]}$.

\section{Summary}

Autocrine and paracrine PDGF signaling contribute to several hallmarks of human cancers, including self-sufficient tumor growth, angiogenesis, stromal recruitment, and tumor invasion and metastasis to distant organs. Although inhibition of PDGF signaling holds promises for treatments of human cancers with activated PDGF signaling, carefully designed pre-clinical/clinical trials are necessary for the success of targeted therapies against components of this signaling pathway.

Received: 2011-07-22; accepted: 2011-07-29.

\section{References}

[1] Ross R, Glomset J, Kariya B, et al. A platelet-dependent serum factor that stimulates the proliferation of arterial smooth muscle cells in vitro [J]. Proc Natl Acad Sci U S A, 1974,71 (4):1207-1210.

[2] Kohler N, Lipton A. Platelets as a source of fibroblast growthpromoting activity [J]. Exp Cell Res, 1974,87(2):297-301.

[3] Ross R, Vogel A. The platelet-derived growth factor [J]. Cell, 1978, 14(2):203-210

[4] Waterfield MD, Scrace GT, Whittle N, et al. Platelet-derived growth factor is structurally related to the putative transforming protein p28sis of simian sarcoma virus [J]. Nature, 1983,304 (5921):35-39.

[5] Doolittle RF, Hunkapiller MW, Hood LE, et al. Simian sarcoma virus onc gene, $v$-sis, is derived from the gene (or genes) encoding a platelet-derived growth factor [J]. Science, 1983,221(4607):275-277.

[6] Hermanson M, Funa K, Hartman M, et al. Platelet-derived growth factor and its receptors in human glioma tissue: expression of messenger RNA and protein suggests the presence of autocrine and paracrine loops [J]. Cancer Res, 1992,52(11):3213-3219.

[7] Smits A, Funa K, Vassbotn FS, et al. Expression of plateletderived growth factor and its receptors in proliferative disorders of fibroblastic origin [J]. Am J Pathol, 1992,140(3):639-648.

[8] Seymour L, Dajee D, Bezwoda WR. Tissue platelet derivedgrowth factor (PDGF) predicts for shortened survival and treatment failure in advanced breast cancer [J]. Breast Cancer Res Treat, 1993,26(3):247-252.

[9] Jechlinger M, Grunert S, Tamir $\mathrm{IH}$, et al. Expression profiling of epithelial plasticity in tumor progression [J]. Oncogene, 2003,22(46):7155-7169.

[10] Jechlinger M, Sommer A, Moriggl R, et al. Autocrine PDGFR signaling promotes mammary cancer metastasis [J]. J Clin Invest, 2006,116(6): 1561-1570.

[11] Ostman A. PDGF receptors-mediators of autocrine tumor growth and regulators of tumor vasculature and stroma [J]. Cytokine Growth Factor Rev, 2004,15(4):275-286.

[12] Andrae J, Gallini R, Betsholtz C. Role of platelet-derived growth factors in physiology and medicine [J]. Genes Dev, 2008,22(10): 1276-1312.

[13] Nister M, Libermann TA, Betsholtz C, et al. Expression of messenger RNAs for platelet-derived growth factor and transforming growth factor-alpha and their receptors in human malignant glioma cell lines [J]. Cancer Res, 1988,48(14):39103918.

[14] Hermanson M, Funa K, Koopmann J, et al. Association of loss of heterozygosity on chromosome $17 p$ with high plateletderived growth factor alpha receptor expression in human malignant gliomas [J]. Cancer Res, 1996,56(1):164-171.

[15] Liu KW, Feng $\mathrm{H}$, Bachoo $\mathrm{R}$, et al. SHP-2/PTPN11 mediates gliomagenesis driven by PDGFRA and INK4A/ARF aberrations in mice and humans [J]. J Clin Invest, 2011,121(3):905-917.

[16] Clarke ID, Dirks PB. A human brain tumor-derived PDGFRalpha deletion mutant is transforming [J]. Oncogene, 2003,22 (5):722-733.

[17] Ozawa T, Brennan CW, Wang L, et al. PDGFRA gene rearrangements are frequent genetic events in PDGFRAamplified glioblastomas [J]. Genes Dev, 2010,24(19):2205 2218.

[18] Liu KW, Hu B, Cheng SY. Platelet-derived growth factor receptor alpha in gliomas: a bad seed [J]. Chin $\mathrm{J}$ Cancer, 2011, 30(9): 590-602.

[19] Pech M, Gazit A, Arnstein P, et al. Generation of fibrosarcomas in vivo by a retrovirus that expresses the normal B chain of platelet-derived growth factor and mimics the alternative splice pattern of the $\mathrm{V}$-sis oncogene [J]. Proc Natl Acad Sci U S A, 1989,86(8):2693-2697.

[20] Wang J, Coltrera MD, Gown AM. Cell proliferation in human soft tissue tumors correlates with platelet-derived growth factor B chain expression: an immunohistochemical and in situ hybridization study [J]. Cancer Res, 1994,54(2):560-564.

[21] Tamborini E, Bonadiman L, Greco A, et al. Expression of ligand-activated KIT and platelet-derived growth factor receptor beta tyrosine kinase receptors in synovial sarcoma [J]. Clin Cancer Res, 2004,10(3):938-943

[22] Wang Y, Zhou BP. Epithelial-mesenchymal transition in breast cancer progression and metastasis [J]. Chin J Cancer, 2011, 30 (9): 603-611.

[23] Bruna A, Darken RS, Rojo F, et al. High TGFbeta-Smad activity confers poor prognosis in glioma patients and promotes cell proliferation depending on the methylation of the PDGF-B gene [J]. Cancer Cell, 2007,11(2):147-160.

[24] Simon MP, Pedeutour F, Sirvent N, et al. Deregulation of the platelet-derived growth factor B-chain gene via fusion with collagen gene COL1A1 in dermatofibrosarcoma protuberans and giant-cell fibroblastoma [J]. Nat Genet, 1997,15(1):95-98.

[25] Greco A, Fusetti L, Villa R, et al. Transforming activity of the chimeric sequence formed by the fusion of collagen gene COL1A1 and the platelet derived growth factor b-chain gene in dermatofibrosarcoma protuberans [J]. Oncogene, 1998,17(10): 1313-1319.

[26] Shimizu A, O'Brien KP, Sjoblom T, et al. The dermatofibrosarcoma protuberans-associated collagen type lalpha1/platelet-derived growth factor (PDGF) B-chain fusion gene generates a transforming protein that is processed to functional PDGF-BB [J]. Cancer Res, 1999,59(15):3719-3723. 
[27] Baxter EJ, Hochhaus A, Bolufer P, et al. The $t(4 ; 22)(q 12 ; q 11)$ in atypical chronic myeloid leukaemia fuses BCR to PDGFRA [J]. Hum Mol Genet, 2002,11(12):1391-1397.

[28] Cools J, DeAngelo DJ, Gotlib J, et al. A tyrosine kinase created by fusion of the PDGFRA and FIP1L1 genes as a therapeutic target of imatinib in idiopathic hypereosinophilic syndrome [J]. N Engl J Med, 2003,348(13):1201-1214.

[29] Golub TR, Barker GF, Lovett M, et al. Fusion of PDGF receptor beta to a novel ets-like gene, tel, in chronic myelomonocytic leukemia with $\mathrm{t}(5 ; 12)$ chromosomal translocation [J]. Cell, 1994,77(2):307-316.

[30] Heinrich MC, Corless CL, Duensing A, et al. PDGFRA activating mutations in gastrointestinal stromal tumors [J]. Science, 2003,299(5607):708-710.

[31] Hirota S, Ohashi A, Nishida T, et al. Gain-of-function mutations of platelet-derived growth factor receptor alpha gene in gastrointestinal stromal tumors [J]. Gastroenterology, 2003,125 (3):660-667.

[32] Abramsson A, Lindblom P, Betsholtz C. Endothelial and nonendothelial sources of PDGF-B regulate pericyte recruitment and influence vascular pattern formation in tumors [J]. $\mathrm{J}$ Clin Invest, 2003,112(8): 1142-1151.

[33] Guo P, Hu B, Gu W, et al. Platelet-derived growth factor-B enhances glioma angiogenesis by stimulating vascular endothelial growth factor expression in tumor endothelia and by promoting pericyte recruitment [J]. Am J Pathol, 2003,162(4): 1083-1093.

[34] Furuhashi M, Sjoblom T, Abramsson A, et al. Platelet-derived growth factor production by B16 melanoma cells leads to increased pericyte abundance in tumors and an associated increase in tumor growth rate [J]. Cancer Res, 2004,64(8): 2725-2733.

[35] Hermansson M, Nister M, Betsholtz C, et al. Endothelial cell hyperplasia in human glioblastoma: coexpression of mRNA for platelet-derived growth factor (PDGF) $\mathrm{B}$ chain and PDGF receptor suggests autocrine growth stimulation [J]. Proc Natl Acad Sci U S A, 1988,85(20):7748-7752.
[36] Hwang RF, Yokoi K, Bucana CD, et al. Inhibition of plateletderived growth factor receptor phosphorylation by STI571 (Gleevec) reduces growth and metastasis of human pancreatic carcinoma in an orthotopic nude mouse model [J]. Clin Cancer Res, 2003,9(17):6534-6544.

[37] Apte SM, Fan D, Killion JJ, et al. Targeting the platelet-derived growth factor receptor in antivascular therapy for human ovarian carcinoma [J]. Clin Cancer Res, 2004,10(3):897-908.

[38] Uehara H, Kim SJ, Karashima T, et al. Effects of blocking platelet-derived growth factor-receptor signaling in a mouse model of experimental prostate cancer bone metastases [J]. J Natl Cancer Inst, 2003,95(6):458-470.

[39] Liu $Q$, Jernigan D, Zhang $Y$, et al. Implication of plateletderived growth factor alpha-receptor in prostate cancer skeletal metastasis [J]. Chin J Cancer, 2011, 30(9): 612-619.

[40] Skobe M, Fusenig NE. Tumorigenic conversion of immortal human keratinocytes through stromal cell activation [J]. Proc Natl Acad Sci U S A, 1998,95(3):1050-1055.

[41] Dong J, Grunstein J, Tejada M, et al. VEGF-null cells require PDGFR alpha signaling-mediated stromal fibroblast recruitment for tumorigenesis [J]. EMBO J, 2004,23(14):2800-2810.

[42] Pietras K, Pahler J, Bergers G, et al. Functions of paracrine PDGF signaling in the proangiogenic tumor stroma revealed by pharmacological targeting [J]. PLoS Med, 2008,5(1):e19.

[43] Heuchel R, Berg A, Tallquist M, et al. Platelet-derived growth factor beta receptor regulates interstitial fluid homeostasis through phosphatidylinositol-3' kinase signaling [J]. Proc Natl Acad Sci U S A, 1999,96(20):11410-11415.

[44] Pietras K, Ostman A, Sjoquist M, et al. Inhibition of plateletderived growth factor receptors reduces interstitial hypertension and increases transcapillary transport in tumors [J]. Cancer Res, 2001,61(7):2929-2934.

[45] Pietras K, Stumm M, Hubert M, et al. STI571 enhances the therapeutic index of epothilone $B$ by a tumor-selective increase of drug uptake [J]. Clin Cancer Res, 2003,9(10 Pt 1):37793787. 